Journal of

\section{Synchrotron}

Radiation

ISSN 0909-0495

Received 20 July 2012

Accepted 18 October 2012

\title{
A simultaneous multiple angle-wavelength dispersive $X$-ray reflectometer using a bent-twisted polychromator crystal
}

\author{
Tadashi Matsushita, ${ }^{\mathrm{a} *}$ Etsuo Arakawa, ${ }^{\mathrm{b}}$ Wolfgang Voegeli ${ }^{\mathrm{a}}$ and Yohko F. Yano ${ }^{\mathrm{c}}$ \\ aPhoton Factory, Institute of Materials Structure Science, KEK, Tsukuba, Ibaraki, Japan, ${ }^{\mathbf{b}}$ Department \\ of Physics, Tokyo Gakugei University, Koganei, Tokyo, Japan, and ${ }^{\mathbf{c}}$ Department of Physics, Kinki \\ University, Higashiosaka, Osaka, Japan. E-mail: matsus@post.kek.jp
}

\begin{abstract}
An X-ray reflectometer has been developed, which can simultaneously measure the whole specular X-ray reflectivity curve with no need for rotation of the sample, detector or monochromator crystal during the measurement. A benttwisted crystal polychromator is used to realise a convergent X-ray beam which has continuously varying energy $E$ (wavelength $\lambda$ ) and glancing angle $\alpha$ to the sample surface as a function of horizontal direction. This convergent beam is reflected in the vertical direction by the sample placed horizontally at the focus and then diverges horizontally and vertically. The normalized intensity distribution of the reflected beam measured downstream of the specimen with a two-dimensional pixel array detector (PILATUS 100K) represents the reflectivity curve. Specular X-ray reflectivity curves were measured from a commercially available silicon (100) wafer, a thin gold film coated on a silicon single-crystal substrate and the surface of liquid ethylene glycol with data collection times of 0.01 to $1000 \mathrm{~s}$ using synchrotron radiation from a bendingmagnet source of a $6.5 \mathrm{GeV}$ electron storage ring. A typical value of the simultaneously covered range of the momentum transfer was $0.01-0.45 \AA^{-1}$ for the silicon wafer sample. The potential of this reflectometer for time-resolved $\mathrm{X}$-ray studies of irreversible structural changes is discussed.
\end{abstract}

Keywords: X-ray reflectivity curve; time-resolved measurement; bent-twisted polychromator crystal; simultaneous measurement.

\section{Introduction}

Specular X-ray reflectometry is an established structural probe for characterizing the surfaces and interfaces of materials (Daillant \& Gibaud, 1999). By analyzing X-ray reflectivity curves, it is possible to determine the thickness, electron density distribution along the surface normal, and surface and interfacial roughness of thin films and multilayers. A popular method of measuring X-ray reflectivity curves is the angledispersive method using collimated and monochromatic $\mathrm{X}$-rays, where the reflected intensity measurement is repeated many times by successively changing the glancing angle of $\mathrm{X}$-rays to the sample surface. Another method is the energydispersive method, where a white X-ray beam is used in combination with an energy-dispersive solid state detector with a fixed glancing angle of X-rays. The data collection time for the angle-dispersive method is typically minutes to tens of minutes even using intense synchrotron radiation and that for the energy-dispersive method is seconds to minutes (Bhattacharya et al., 2003). Until recently, most time-resolved X-ray reflectivity measurements have been limited to time scales of minutes to hours (Richter et al., 1998; Rossi Abertini et al., 2003; Generosi et al., 2005; Paci et al., 2005, 2006; GonzalezSilveria et al., 2007; Yano et al., 2009). Millisecond time-resolution X-ray reflectometers were proposed (White et al., 1999; Garrett et al., 2001), in which white X-rays reflected by the sample are simultaneously wavelength-dispersed by single or multiple crystal analyzer(s). An exceptionally high time resolution of $100 \mathrm{ps}$ was achieved recently by Nüske et al. (2011) in a pump-probe mode with the angle-dispersive method. However, in this pump-probe mode the structural changes of the sample must be repeated many thousands of times at every glancing angle position of the sample, thus this method is not applicable to samples in which structural changes are irreversible or not easily repeatable. For studying the structural kinematics or dynamics of such samples, it would be better to be able to simultaneously measure the whole profile of the X-ray reflectivity curve and to successively measure the time-dependent changes after applying a stimulus to the specimen.

In previous studies (Matsushita et al., 2008, 2009, 2010) we reported a method of simultaneously measuring specular 
$\mathrm{X}$-ray reflectivity curves in milliseconds to seconds with no need for angle scanning of the sample, detector or polychromator crystal during measurement. In this method the reflectivity curve is measured with a position-sensitive detector as a function of X-ray energy using a convergent $\mathrm{X}$-ray beam which has a one-to-one correspondence between direction and energy. Although the data collection time was short, the practically measured range of the perpendicular momentum transfer was not wide enough because the X-ray energy range was limited. Moreover, the minimum detectable reflectivity was also limited to approximately $10^{-6}$ because of the read-out noise of the X-ray CCD detector.

In this study we report improvements of the method to widen the simultaneously covered range of the perpendicular momentum transfer, $Q$, and to lower the detectable minimum reflectivity. For widening the $Q$-range, we used a convergent $\mathrm{X}$-ray beam for which both the energy $E$ and the glancing angle $\alpha$ to the sample surface change continuously as a function of direction. We kept the geometry such that the sample surface was stationary in the horizontal plane in order to be able to study liquid surfaces. For lowering the detectable minimum reflectivity, we used a photon-counting pixel array detector with no read-out noise and a very sharp point-spread function.

\section{Principle of the method}

\subsection{X-ray optics and simultaneously covered range of momentum transfer}

A specular X-ray reflectivity curve is a plot of the X-ray reflectivity as a function of the perpendicular momentum transfer $Q$ which is defined by

$$
Q=4 \pi \sin \alpha / \lambda=4 \pi \sin \alpha E / h c,
$$

where $\alpha$ is the glancing angle of $\mathrm{X}$-rays, $\lambda$ is the wavelength, $E$ is the energy, $h$ is the Planck constant and $c$ is the speed of light.

The geometry of the present method is schematically shown in Fig. 1. The main component of the X-ray optics is the curved crystal polychromator in the reflection geometry. The diffracting plane of the polychromator crystal is assumed to be parallel to the surface. A synchrotron white X-ray beam from a bending-magnet source is incident on the polychromator crystal. The horizontal and vertical beamline slits are kept wide open to guide an X-ray beam having a rectangular cross section $A_{0} B_{0} C_{0} D_{0}$ into the experimental hutch. An inclined slit is placed upstream of the polychromator crystal so that a beam through one end of the slit aperture hits the upper-right corner of the polychromator crystal at $A_{1}$, which is slightly (1-3 $\mathrm{mm})$ above the electron orbit plane of the storage ring by an amount $\Delta h_{A 1}$, and a beam through the other end of the slit aperture hits the lower-left corner of the crystal at $C_{1}$, which is below the electron orbit plane by an amount $\Delta h_{C 1}$ (10-15 mm).

If the crystal is ellipsoidally bent such that the X-ray source $S$ and the focal point $F_{\mathrm{S}}$ are located at its two foci, all the X-ray

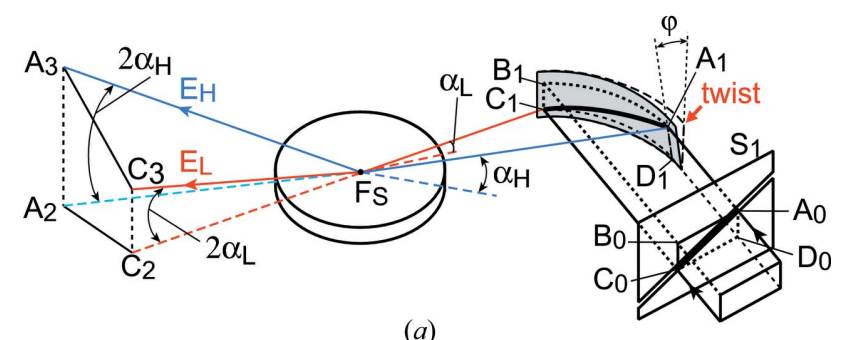

(a)

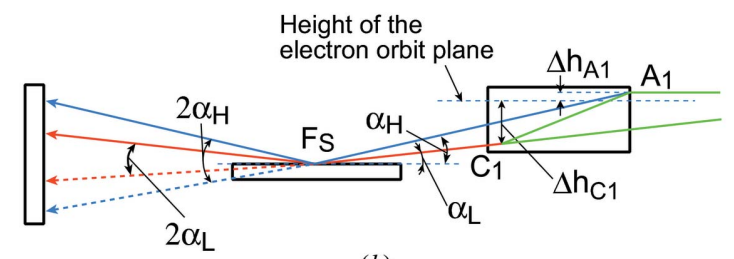

(b)

Figure 1

Bird's eye $(a)$ and side $(b)$ views of the geometry of the simultaneous multiple angle-wavelength dispersive X-ray reflectometer. The crystal is bent horizontally to realise horizontal focusing and then twisted in such a way that the surface normal along the straight line $A_{1} D_{1}$ is inclined downward by an angle $\varphi$, while that along $B_{1} C_{1}$ stays horizontal in the original direction. $\alpha_{\mathrm{H}}$ is the glancing angle of the ray along the path $A_{1} F_{\mathrm{S}}$ to the sample surface at $F_{\mathrm{S}}$, and $\alpha_{\mathrm{L}}$ is that for the ray along the path $C_{1} F_{\mathrm{S}}$. The X-ray beam is specularly reflected by a sample placed horizontally at $F_{\mathrm{S}}$, then horizontally diverges directed toward points on the line $A_{3} C_{3}$ on the detector surface.

beams reflected by the crystal are directed toward the focus $F_{\mathrm{S}}$. However, we do not use a crystal with an ellipsoidal shape because it is difficult to bend the crystal to an ellipsoidal shape. Instead, we used a bent and twisted crystal. We first calculated the surface normal directions along the curve $A_{1} C_{1}$ of an ellipsoid. Next, we considered a surface that consists of the tangents to a generating line of the ellipsoid at each point of the curve $A_{1} C_{1}$. The resulting surface is a train of almost vertical straight lines with gradually changing direction that touch the ellipsoid on the curve $A_{1} C_{1}$. We then prepared concave and convex bronze blocks which mimic such a surface. The thin crystal is sandwiched between these convex and concave shaped surfaces, as is shown in Fig. 2. An X-ray beam having infinitesimally small vertical width incident exactly on the curve $A_{1} C_{1}$ is focused at $F_{\mathrm{S}}$. If the vertical width of the inclined slit $S_{1}$ upstream of the polychromator crystal is finite,

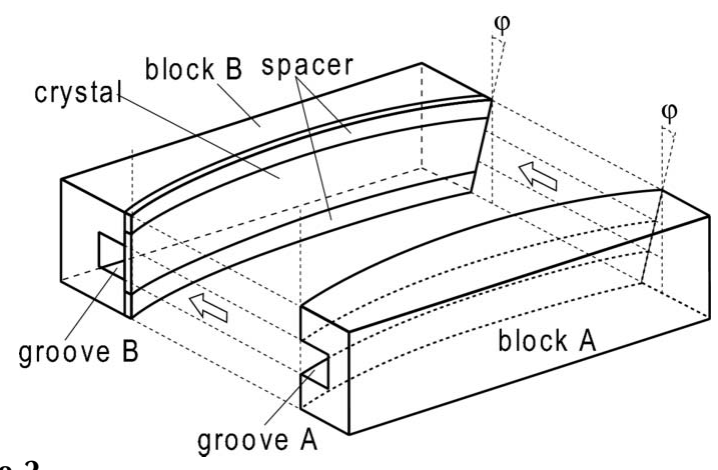

Figure 2

A bender used to create the bent-twisted crystal polychromator. The crystal was bent and twisted by sandwiching it between two water-cooled bronze blocks which have preconfigured convex and concave surfaces. 
but small enough, $\mathrm{X}$-ray beams reflected at points near the curve $A_{1} C_{1}$ are horizontally focused and vertically condensed to a tiny spot at $F_{\mathrm{S}}$. The direction of the surface normal at any point along the straight line $A_{1} D_{1}$ is inclined downward by an angle $\varphi$, while that at any point along the vertical line $B_{1} C_{1}$ stays horizontal. The beam diffracted at $C_{1}$ keeps the original vertical deflection angle and is directed toward $F_{\mathrm{S}}$. The vertical deflection angle from the horizontal plane of the beam along the line $C_{1} F_{\mathrm{S}}$ is the same as that of the beam along $C_{0} C_{1}$ and is given by

$$
\alpha_{\mathrm{L}}=\Delta h_{C 1} / p .
$$

Here, $p$ is the distance from the polychromator crystal to the $\mathrm{X}$-ray source. The beam diffracted at $A_{1}$ is deflected downward by an angle $\delta_{\mathrm{H}}$ defined by

$$
\delta_{\mathrm{H}}=2 \varphi \sin \theta,
$$

where $\theta$ is the Bragg angle at $A_{1}$. The angle between the line $A_{1} F_{\mathrm{S}}$ and the horizontal plane is given by

$$
\alpha_{\mathrm{H}}=\left(\Delta h_{A 1}+\Delta h_{C 1}\right) / q_{A 1}+\alpha_{\mathrm{L}},
$$

where $q_{A 1}$ is the distance from the focus $F_{\mathrm{S}}$ to point $A_{1}$. By setting the twist angle $\varphi$ of the crystal to realise the relation $\delta_{\mathrm{H}}=\alpha_{\mathrm{H}}$, the beam reflected at $A_{1}$ is directed toward $F_{\mathrm{S}}$. At the same time the beam diffracted at an arbitrary point $P$ (not shown in Fig. 1) along $A_{1} C_{1}$ is also directed toward $F_{\mathrm{S}}$. The beam represented by the line $A_{0} C_{0}$ is horizontally focused and vertically condensed at $F_{\mathrm{S}}$ after being reflected by the crystal.

The sample is placed almost horizontally at $F_{\mathrm{s}}$. The X-ray beam is specularly reflected in the vertical direction. The glancing angle $\alpha$ of each ray of the convergent X-ray beam continuously varies between $\alpha_{\mathrm{L}}$ and $\alpha_{\mathrm{H}}$ depending on its horizontal path direction. Downstream of the focus, the X-ray beam diverges horizontally and directs towards points on the line $A_{3} C_{3}$ on the detector surface. At the same time, the energy $E$ (wavelength $\lambda$ ) of the X-ray beam reflected at $P$ also continuously varies between $E_{\mathrm{H}}$ and $E_{\mathrm{L}}$ along the line $A_{1} C_{1}$, where $E_{\mathrm{H}}$ and $E_{\mathrm{L}}$ are the energies of the X-ray beams monochromated at $A_{1}$ and $C_{1}$, respectively.

The perpendicular momentum transfer for the ray from $P$ to $F_{\mathrm{S}}$ is given by equation (1), resulting in a continuous change of the perpendicular momentum transfer along $A_{3} C_{3} . \alpha$ and $E$ can be experimentally determined as will be described in $\S 3.1$. When the sample is removed, the X-ray beam hits points on the line $A_{2} C_{2}$ on the detector surface. By normalizing the reflected intensity distribution $I$ measured along the line $A_{3} C_{3}$ by the intensity distribution $I_{0}$ along the line $A_{2} C_{2}$, the X-ray reflectivity curve profile is obtained without any mechanical movement of the sample, detector or polychromator crystal.

\subsection{Resolution}

The momentum transfer resolution of the system at the $\mathrm{X}$-ray energy $E$ is dependent on the energy spread $\delta E$ and the vertical angular spread $\delta \alpha$ of the X-ray beam detected by each pixel element of the detector. The spread in the momentum transfer caused by the energy spread of the X-ray beam is given by the relation $\delta Q_{E} / Q=\delta E / E$. The latter is simply given by $\delta Q / Q=\delta \alpha \cot \alpha$, where $\alpha$ is the angle between the convergent X-ray beam and the surface of the sample.

The energy spread can be characterized into the four contributions $\delta E_{1}, \delta E_{2}, \delta E_{3}$ and $\delta E_{4}$, which come from (1) the horizontal size of the source, (2) the size of a detector pixel, (3) the thickness of the polychromator crystal and (4) the intrinsic angular width of diffraction, respectively. The contribution from the thickness of the crystal comes from the fact that the X-ray beam penetrates into the crystal and is diffracted within the crystal. These four factors can roughly be estimated by simple ray-tracing calculations. When we assume a $0.3 \mathrm{~mm}$-thick Si (111) crystal in the reflection geometry, $p=$ $20.5 \mathrm{~m}$, crystal-to-focus distance $=0.43 \mathrm{~m}$, a detector pixel size of $0.172 \mathrm{~mm}$, specimen-to-detector distance $=580 \mathrm{~mm}$ and horizontal size of the source $=3.0 \mathrm{~mm}$, it is roughly estimated that $\delta E_{1} \simeq 13 \mathrm{eV}, \delta E_{2} \simeq 15 \mathrm{eV}, \delta E_{3} \simeq 5 \mathrm{eV}$ and $\delta E_{4} \simeq 3 \mathrm{eV}$ at $\sim 19 \mathrm{keV}$. The total energy spread $\delta E=\left(\delta E_{1}{ }^{2}+\delta E_{2}{ }^{2}+\delta E_{3}{ }^{2}+\right.$ $\left.\delta E_{4}^{2}\right)^{1 / 2}$ can be estimated to be approximately $21 \mathrm{eV}$, resulting in $\delta Q_{E} / Q=\delta E / E=1.1 \times 10^{-3}$.

Two factors should be considered regarding the angular resolution. One is the angular divergence $\delta \alpha_{1}$ of the X-ray beam of an infinitely small horizontal width. Since the surface normal does not change along a vertical line of the polychromator crystal, the angular divergence of this X-ray beam is determined by the vertical width of the slit $\delta_{\mathrm{w}}$, the vertical size $\delta_{\mathrm{sv}}$ of the source and the source-to-slit distance $p_{\mathrm{S}-\mathrm{s}}$. If $\delta_{\mathrm{w}}=$ $0.1 \mathrm{~mm}, \delta_{\mathrm{sv}}=0.2 \mathrm{~mm}$ and $p_{\mathrm{S}-\mathrm{S}}=20.5 \mathrm{~m}, \delta \alpha_{1}$ is estimated to be $1.1 \times 10^{-3}$ degrees $\left(2 \times 10^{-5}\right.$ radians $)$, which is sufficiently small. Another factor is the scattering angle covered by a single pixel element of the detector, which is given by

$$
\delta \alpha_{2}=\Delta \alpha / n,
$$

where $\Delta \alpha=\alpha_{\mathrm{H}}-\alpha_{\mathrm{L}}$, and $n$ is the number of pixel elements which are required to cover this angle range. If $\Delta \alpha$ is $1.5^{\circ}$ and $n$ is 150 , the angle resolution is $0.01^{\circ}\left(1.75 \times 10^{-4} \mathrm{rad}\right)$. This gives a resolution $\delta Q_{\alpha} / Q=\delta \alpha_{2} \cot \alpha$. For $\alpha=1.0^{\circ}$, this value is 0.01 .

With such estimations it is understood that the resolution of the momentum transfer is mainly governed by the angular resolution of the system in the present geometry.

\subsection{Intensity of the reflected $X$-ray beam}

The intensity of the synchrotron X-ray beam diffracted at $C_{1}$ is several orders of magnitude weaker than that at $A_{1}$ because the height at $A_{1}$ is much closer to that of the electron orbit plane than the height at $C_{1}$ as is shown in Fig. 1(b). The intensity of the diffracted X-ray beam along the line $A_{1} C_{1}$ is given in Fig. 3, where the abscissa indicates the angle of the ray from a point on the line $A_{1} C_{1}$ to $F_{\mathrm{S}}$. Since the energy $\left(E_{\mathrm{H}}\right)$ of the beam diffracted at $A_{1}$ is only $15-20 \%$ higher than that $\left(E_{\mathrm{L}}\right)$ of the beam diffracted at $C_{1}$ in the present geometry, as will be described in \$3.1, we neglect the energy dependences of the bending-magnet radiation intensity, the Bragg reflectivity and the sensitivity of the detector in the semi-quantitative discussion of the observed reflected intensity. $I_{0}$ is the intensity distribution of the X-ray beam after reflection by the poly- 


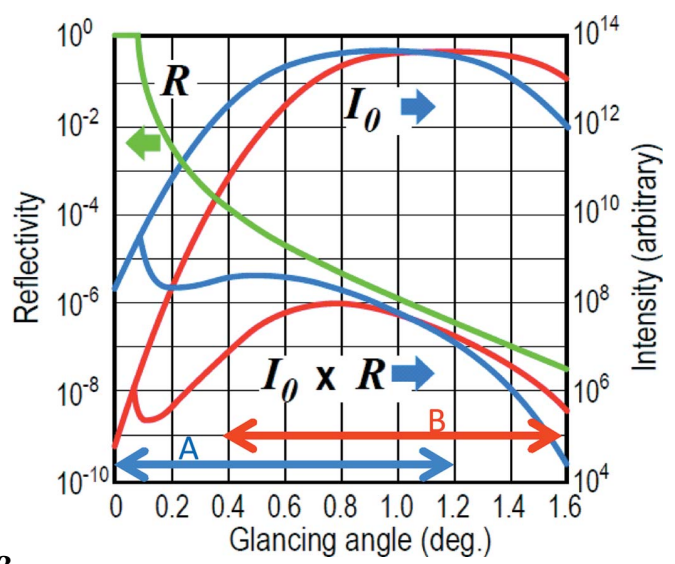

Figure 3

A semi-quantitative representation of the distribution of the specularly reflected beam intensity as a function of the glancing angle of the X-ray beam to the sample surface. $I_{0}$ : intensity distribution of the convergent $\mathrm{X}$-ray beam incident onto the sample. Blue and red lines are for cases $\alpha_{0}=$ $0.96^{\circ}$ and $\alpha_{0}=1.20^{\circ}$, where $\alpha_{0}$ is the glancing angle which the horizontal $\mathrm{X}$-ray beam from the source to the polychromator crystal makes with the sample surface after being reflected downward by the polychromator crystal. $R$ : the specular reflectivity of a sample (silicon single crystal). $I_{0} \times R$ : the product of $I_{0}$ and $R$ which approximately represents the intensity distribution $I$ of the X-ray beam specularly reflected by the sample.

chromator crystal for two cases, $\alpha_{0}=0.96^{\circ}$ or $1.20^{\circ}$. Here, $\alpha_{0}$ is the glancing angle that the horizontal X-ray beam from the source to the polychromator crystal makes with the sample surface after being reflected downward by the polychromator crystal. $I_{0}$ is proportional to the vertical intensity distribution along the line connecting $A_{1}$ to $D_{1}$ within the approximation described above. $I_{0}$ is calculated using the calculation code SPECTRA (Tanaka \& Kitamura, 2001) for the case of the bending-magnet radiation of the $6.5 \mathrm{GeV}$ ring (horizontal emittance $=294 \mathrm{nmrad}$ ) of the Photon Factory. The value of $\alpha_{0}$ can be controlled by adjusting the vertical inclination of the polychromator crystal. The covered glancing angle range for the beams from $C_{1}$ to $A_{1}$ is shown by the two-way arrows $A$ and $B$ for these two cases. The positions shown by the left ends of the two-way arrows $A$ and $B$ correspond to the glancing angles of the X-ray beams through paths $C_{1} F_{\mathrm{S}}$ and the right ends to those of the X-ray beams through the path $A_{1} F_{\mathrm{S}}$. The curve $R$ is the specular reflectivity of the sample. The specular reflectivity of the sample for the beam along $A_{1} F_{\mathrm{S}}$ is several orders of magnitude lower than that for the beam along $C_{1} F_{\mathrm{S}}$. The reflected beam intensity distribution along $A_{3} C_{3}$ on the detector surface is proportional to the product $I_{0} \times R$. Note that the difference between maximum and minimum intensities of the curve $I_{0} \times R$ is within only three or four orders of magnitude in spite of the difference of seven to eight orders of magnitude in $R$. This makes time-resolved measurements much easier.

\section{Experiments}

\subsection{X-ray optics}

As a polychromator crystal, a silicon (111) wafer of size $200 \mathrm{~mm} \times 20 \mathrm{~mm} \times 0.3 \mathrm{~mm}$ was used in the reflection geometry. To bend and twist the crystal, we prepared two directly water-cooled bronze metal blocks having convex and concave surfaces as discussed in $\$ 2.1$ and shown in Fig. 2. Moreover, we made grooves in the middle parts of these bronzes: groove $A$ was made to secure incident and reflected $\mathrm{X}$-ray beam paths and groove $B$ was to remove any scattering materials right behind the crystal and to lower the background intensity. The crystal was sandwiched between these convex and concave surfaces with spacers to reduce clamping forces acting on the crystal as shown in Fig. 2. The thermal contact between the bronze blocks and the crystal was kept by using liquid gallium-indium alloy. Without the water cooling or in the case where the water cooling was insufficient, the shape of the diffracted X-rays was deformed suggesting thermal deformation of the crystal. The shapes of these concave and convex surfaces were designed and configured so as to cause horizontal focusing and vertical condensing of reflected X-rays at $430 \mathrm{~mm}$ from the center of the crystal when the source-tocrystal distance is $20.5 \mathrm{~m}$ and the Bragg angle for the central beam is $6.28^{\circ}$. The slit upstream of the polychromator crystal was $1.0 \mathrm{~mm}$ wide and was inclined $36.4^{\circ}$ from the horizontal line, so the shape of the X-ray beam incident on the polychromator crystal is the diagonal line of a rectangle $[16.6 \mathrm{~mm}$ $(\mathrm{H}) \times 12.2 \mathrm{~mm}(\mathrm{~V})]$. Downstream of the polychromator, the shape of the X-ray beam was a slightly curved and inclined line with a width of $1 \mathrm{~mm}$. An inclined straight-line slit with a width of $0.1 \mathrm{~mm}$ was placed downstream of the polychromator crystal so that the intensity distribution of the X-ray beam incident on the sample is along a linear line downstream of this slit. A slit and a helium beam path covered with lead sheets were also placed downstream of the sample to reduce background intensities.

The beam size at the focus position was observed by a twodimensional pixel array detector [PILATUS 100K (Kraft et al., 2009)] with appropriate beam absorbers which made the intensity distribution along the inclined line relatively flat in order to bring the intensities of any parts of the X-ray beam within the dynamic range of the detector and to image the focus with relatively equal contributions from different energy components. By such an observation, the horizontal focus size was measured to be $0.8 \mathrm{~mm}$ at a distance of $430 \mathrm{~mm}$ from the center of the polychromator crystal. The vertical X-ray beam spot size was measured to be $0.2 \mathrm{~mm}$ also at a distance of $430 \mathrm{~mm}$ by vertically scanning a knife edge with the PILATUS detector located $580 \mathrm{~mm}$ downstream of the focus and differentiating the obtained intensity distribution.

To measure the intensity distribution of the specularly reflected beam, the PILATUS $100 \mathrm{~K}$ detector was placed $580 \mathrm{~mm}$ downstream of the specimen. The sensitive area of the detector is $83.8 \mathrm{~mm} \times 33.5 \mathrm{~mm}$ and the size of a pixel element is $172 \mu \mathrm{m} \times 172 \mu \mathrm{m}$. The energy of the X-ray beam was calibrated by observing the positions of absorption edges at the detector surface when several kinds of metal foils were placed at the focus position in a similar manner as reported earlier (Matsushita et al., 2007). The energy range was from around $16 \mathrm{keV}$ to $21 \mathrm{keV}$ along the line $A_{1} C_{1}$ of the polychromator crystal shown in Fig. 1. For example, in the case of 
the silicon single-crystal sample described in $\$ 3.2$ and $\$ 4.1$ the $\mathrm{X}$-ray energy varied from $16.9 \mathrm{keV}$ to $20.3 \mathrm{keV}$. The energy spread covered by one pixel was estimated to be $24.1 \mathrm{eV}$ by dividing the whole covered energy range $(16.9 \mathrm{keV}$ to $20.3 \mathrm{keV}$ ) by the number (141) of pixels to cover this energy range.

The position of the reflected beam was easily recorded by the detector without using any absorber, while the position of the direct beam without the specimen was recorded with several absorbers in order to avoid saturation of pixel elements of the detector. With such measurements, the vertical separation between the reflected and direct beams was measured as a function of the horizontal position on the detector surface. By dividing halves of these vertical separations with the distance between the X-ray beam focus and the detector, glancing angles of the X-ray beam were determined as a function of the horizontal position on the detector surface. In the case of silicon crystal samples (\$3.2), the glancing angle varied from $0.033^{\circ}$ to $1.24^{\circ}$ within the convergence of the $\mathrm{X}$-ray beam.

\subsection{Samples}

Samples were placed almost horizontally at the focus position. The glancing angle of the X-ray beam could be controlled by rotating the sample around the horizontal rotation axis except in the case of liquid samples. As samples, we used a commercially available mechano-chemically polished (100) silicon wafer, a $15.4 \mathrm{~nm}$-thick gold film coated on a silicon single-crystal substrate and liquid ethylene glycol. The mechano-chemically polished silicon sample was studied to check whether a wide $Q$-range could be covered simultaneously and to determine the detectable minimum reflectivity. The gold film sample was used in order to check whether Kiessig fringes (Kiessig, 1931) could be observed. The size of the substrate was $15 \mathrm{~mm} \times 10 \mathrm{~mm} \times 0.5 \mathrm{~mm}$. The ethylene glycol sample filled in a shallow $100 \mathrm{~mm}$-diameter circular trough was used to demonstrate that a liquid surface can be studied with the present reflectometer. No anti-vibration devices were installed when the sample was measured.

\subsection{Measurements of intensity and position of specularly reflected X-ray beam}

The intensity distribution $I$ of the reflected beam from the sample was measured by the PILATUS detector placed $580 \mathrm{~mm}$ downstream of the focus. Fig. 4 shows an example of a part of the recorded detector output image of the specularly reflected beam from the silicon wafer sample. The $x$ - and $y$ axes are parallel to the horizontal and vertical directions, respectively. From the $y$-coordinate of the specularly reflected beam, the scattering angle at a particular horizontal position $x$ can be determined. The $x$-coordinate can be converted to $\mathrm{X}$-ray energy from the calibrated curve. From this X-ray energy and the scattering angle, the perpendicular momentum transfer $Q$ was determined following equation (1). The intensity of the specularly reflected beam at each $Q$ was obtained by plotting the intensity profile along the $y$-axis as is (a)

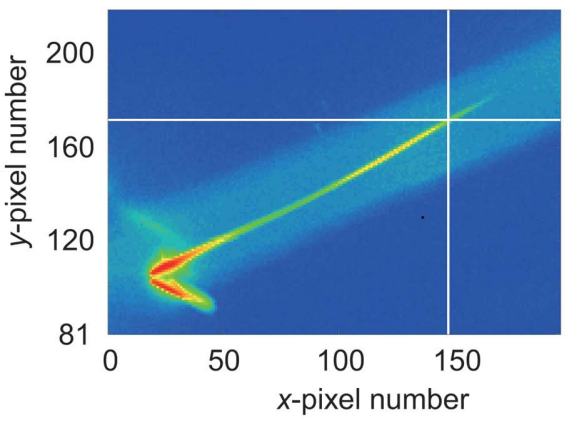

(b)

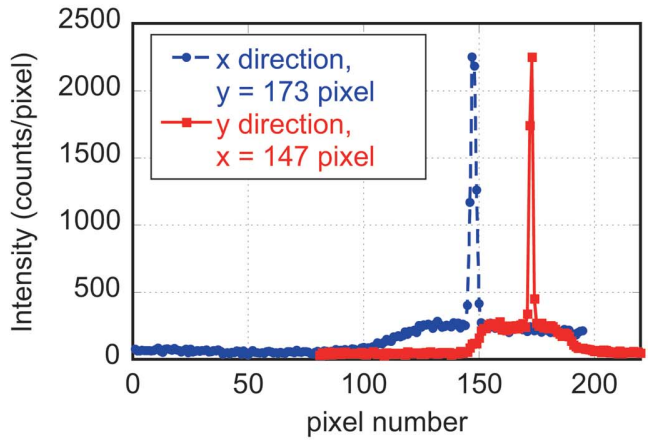

Figure 4

(a) Part of the detector image of the X-ray beam reflected from the silicon single-crystal sample. The $x$-axis is parallel to the horizontal direction across the X-ray beam direction, while the $y$-axis is parallel to the vertical direction. Units on the $x$ - and $y$-axes are pixel numbers of the detector and the size of a pixel is $172 \mu \mathrm{m} \times 172 \mu \mathrm{m}$. The dark parts are shadows of a slit downstream of the sample. The inclined broad and slightly bright band represents X-ray intensity coming through the slit downstream of the sample and this consists of coplanar and non-coplanar diffuse scattering. A much brighter inclined line in the middle of the broad band is the specularly reflected beam. (b) Intensity profiles along white lines parallel to $x$ (blue) and $y$ (red) axes in (a) including a specular reflection at $Q=0.4 \AA^{-1}$.

shown in Fig. 4(b) and then separating the specular reflection intensity from the background intensity. This problem of background subtraction from experimental data will further be discussed in $\$ 5.3$.

\subsection{Determination of the X-ray beam intensity distribution incident to the sample}

To experimentally determine the reflectivity, it is also necessary to measure the intensity distribution $I_{0}$ at the detector when the sample is removed from the X-ray path. The ratio between $I$ and $I_{0}$ gives the reflectivity $R$.

The difference between maximum and minimum intensities in $I_{0}$ is six to seven orders of magnitude. Some pixel elements receiving X-ray beams in the direction near the electron orbit plane are easily saturated or the dead time of the detector becomes serious (Trueb et al., 2012) even when using absorbers to attenuate the beam intensity. Moreover, the contribution from higher harmonics was enhanced when using absorbers, leading to erroneous estimation of $I_{0}$. The following method was used to avoid these problems. In the region where $Q(\alpha)$ is small, the incident beam intensity $I_{0}$ is low but the reflectivity is relatively high as is shown in Fig. 3. Both the reflected and direct beam intensities, $I$ and $I_{0}$, were measured 
without saturation of the detector even with no absorbers. The reflectivity $R$ was determined from measured $I$ and $I_{0}$ in the corresponding horizontal range $\Delta x_{1}$ of the detector. Next, the sample was rotated slightly, with the result that the same $Q$ range was covered on the detector in its horizontal position range $\Delta x_{2}$ adjacent to $\Delta x_{1}$. Since $R$ in this $Q$-range was already determined and the reflected beam intensity $I$ in this range was measured, the incident beam intensity could be determined from the relation $I_{0}=I / R$. A typical count rate of $I$ per pixel element was ten to several thousand per second which was sufficiently low and almost free from saturation of the detector. By repeating this procedure we determined the direct beam intensity distribution $I_{0}$ across the surface of the detector.

\section{Results}

\subsection{Reflectivity curves from a silicon (100) single-crystal wafer}

Fig. 5 shows specular X-ray reflectivity curves from the silicon (100) wafer. Curve $a$ was obtained with a data collection time of $1000 \mathrm{~s}$. The reflectivity curve profile is recorded in the momentum transfer range from 0.01 to $0.45 \AA^{-1}$. Typical signal count rates were 4300,20 and 2.0 counts s$^{-1}$ at $Q=$ $0.02,0.30$ and $0.45 \AA^{-1}$, respectively. The minimum reflectivity in curve $a$ was $6.3 \times 10^{-9}$ at $Q=0.45 \AA^{-1}$. Curves $b-d$ were obtained with data collection times of $10,1.0$ and $0.1 \mathrm{~s}$, respectively.

Curve $e$ was obtained by the angle-scan mode using the present reflectometer setting; the horizontal width of the

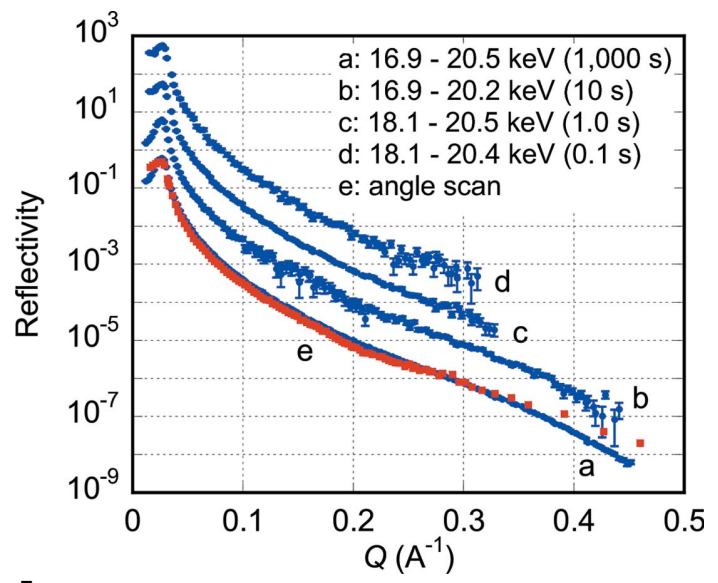

Figure 5

X-ray reflectivity curves from a mechano-chemically polished silicon (100) wafer. Curves $a$ and $b$ were obtained with data collection times of 1000 and $10 \mathrm{~s}$, respectively. Curves $c$ and $d$ were obtained with data collection times of 1.0 and $0.1 \mathrm{~s}$, respectively, after rotating the sample to lower the glancing angle in order to enhance the incident beam intensity $I_{0}$ in the covered $Q$-range to compensate for the short exposure time. Each curve is shifted vertically for clarity. The used X-ray energy ranges are given in the figure. Curve $e$ was obtained by the angle-scan mode using the present reflectometer setting with a horizontal slit of $0.1 \mathrm{~mm}$ width downstream of the polychromator. The angle-scan curve $e$ was obtained by connecting seven partial reflectivity curves measured using seven different X-ray energies $(17.7,18.2,18.6,19.1,19.5,19.9$ and $20.3 \mathrm{keV}$ ). Error bars are shown for all data points of all the curves.
$\mathrm{X}$-ray beam was narrowed to $0.1 \mathrm{~mm}$ with an extra slit downstream of the polychromator and the glancing angle of the X-ray beam was scanned by rotating the sample around a horizontal axis. The vertical scale was taken to be the same as that for the curve $a$. In this measurement the measured $Q$ range was divided into seven regions. We placed a $0.1 \mathrm{~mm}$ wide vertical slit at seven corresponding different horizontal positions to be able to measure the reflected intensity at a reasonable count rate. For example, at low $Q$-range, we placed the slit to select a low-intensity part of the convergent X-ray beam because the reflectivity from the sample is high, while at high $Q$-value we placed the slit to select a high-intensity part because the reflectivity from the sample is low. In this way we collected seven partial profiles of the reflectivity curve with seven slightly different $\mathrm{X}$-ray energies $(17.7,18.2,18.6,19.1$, $19.5,19.9$ and $20.3 \mathrm{keV}$ ) and connected these seven partial reflectivity profiles to make one profile, which is the curve $e$. In other words, the lower $Q$-part of curve $e$ was measured with lower X-ray energies and the higher $Q$-part was measured with higher X-ray energy.

\subsection{Reflectivity curves from a thin gold layer on a silicon single-crystal substrate}

Fig. 6 shows measured reflectivity curves from the $15.4 \mathrm{~nm}$ thick gold film on a silicon substrate. Curve $a$ was obtained with a data collection time of $1000 \mathrm{~s}$. Curves $b, c$ and $d$ were obtained with data collection times of $1.0,0.1$ and $0.01 \mathrm{~s}$, respectively. The central glancing angle of the convergent beam was made at $0.56^{\circ}$ covering the glancing angle range $0.08-1.22^{\circ}$ when curve $a$ was measured. Curve $e$ was obtained with an angle-scan X-ray $(E=15 \mathrm{keV}$ ) reflectometer (Yano et al., 2009) on an undulator beamline at SPring-8 in $644 \mathrm{~s}$ and the origin of its vertical axis is taken to be the same as that of curve $a$.

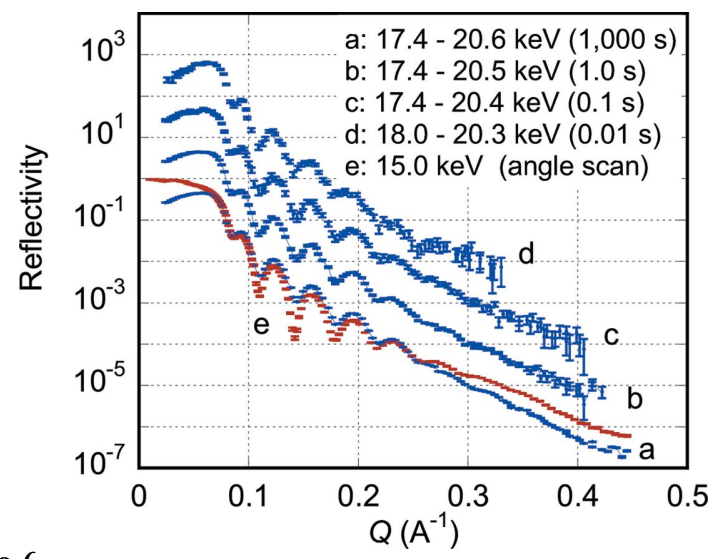

Figure 6

X-ray reflectivity curves from a $15.4 \mathrm{~nm}$-thick gold film on a silicon singlecrystal substrate. Curve $a$ was obtained with a data collection time of $1000 \mathrm{~s}$. Curves $b, c$ and $d$ were obtained with data collection times of 1.0, 0.1 and $0.01 \mathrm{~s}$, respectively. Curves $b, c$ and $d$ are shifted vertically to avoid overlapping each other. The curve $e$ was obtained with an angle-scan X-ray $(E=15 \mathrm{keV})$ reflectometer on an undulator beamline at SPring-8 in $644 \mathrm{~s}$. The origin of its vertical axis is taken to be the same as that of the curve $a$. The used X-ray energy ranges are given in the figure. 


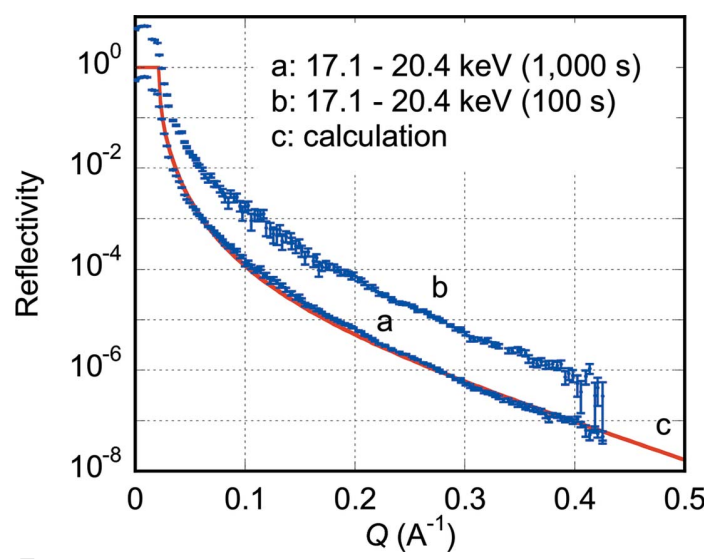

Figure 7

X-ray reflectivity curves from a liquid surface of ethylene glycol. Curves $a$ and $b$ were obtained with data collection times of 1000 and $100 \mathrm{~s}$, respectively. Curve $b$ is vertically shifted for clarity by one order of magnitude. The used X-ray energy ranges are given in the figure. Curve $c$ is a calculated one.

\subsection{Reflectivity curve from a liquid surface}

Curves $a$ and $b$ in Fig. 7 are reflectivity curves from an ethylene glycol liquid surface obtained with data collection times of 1000 and $100 \mathrm{~s}$, respectively. The simultaneously covered range of the momentum transfer was from 0.003 to $0.43 \AA^{-1}$. The minimum reflectivity in curve $a$ is $4.8 \times 10^{-8}$ at $Q=0.43 \AA^{-1}$. Curve $c$ is a calculated reflectivity curve which was obtained in the same manner as in a previous paper (Yano et al., 2010). Curve $b$ is one order of magnitude shifted upward along the $y$-axis for clarity.

\section{Discussion}

\subsection{Width of the simultaneously covered range and resolution of the momentum transfer}

Specular X-ray reflectivity was simultaneously measured from almost zero momentum transfer to over $0.4 \AA^{-1}$ in most curves for the silicon (100) wafer, thin gold film and liquid ethylene glycol samples. The simultaneously covered $Q$-range, $\Delta Q$, is widened if we select a higher $\mathrm{X}$-ray energy since $\Delta Q$ is proportional to the X-ray energy $E$. However, the sensitivity of the PILATUS detector falls off rapidly at high energies. As a compromise, we used X-ray energies around $20 \mathrm{keV}$ in the present study. $\Delta Q$ would also be widened by choosing a geometry that would widen the angle range $\Delta \alpha$ although it is necessary to confirm that sufficient X-ray intensities are available in this angular range.

The largest factor contributing to the resolution of the momentum transfer is the angular width accepted by each pixel element as discussed in \$2.2. This angular width was $0.0084^{\circ}$ since $\Delta \alpha=1.21^{\circ}$ and $n=141$ for the case of the silicon sample, which gives a resolution $\delta Q / Q=0.0092$ at $Q=0.3 \AA^{-1}$. The resolution will be further improved by increasing the pixel number $n$ in (5) using a detector of smaller pixel elements or a larger detector at a larger distance from the sample.

\subsection{Comparison with the angle-scan method}

For the case of the silicon wafer, curve $a$ in Fig. 5 measured with the present dispersive method agrees very well with curve $e$ measured with the angle-scan mode down to a reflectivity of $\sim 2 \times 10^{-7}$. The reason for the small difference between curves $a$ and $e$ below a reflectivity of $\sim 2 \times 10^{-7}$ is not yet clear.

For the case of a gold film on a silicon substrate, curve $a$ in Fig. 6 measured with the present dispersive method agrees fairly well with curve $e$ measured with the angle-scan method down to a reflectivity of $\sim 3 \times 10^{-5}$. The period of the Kiessig fringes of curve $a$ agrees well with that of curve $e$. The fitted thickness of the gold layer was 15.37-15.26 nm for exposure durations ranging from $1000 \mathrm{~s}$ to $0.1 \mathrm{~s}$, while that estimated from the angle-scan curve was $15.39 \mathrm{~nm}$. We assumed a single gold layer on a silicon substrate. The analysis was made after Parratt's recursion formula (Parratt, 1954) by taking into account the energy and the glancing angle change over $Q$ value. The dips of curve $a$ are shallower than those of curve $e$ This is because the resolution is several times poorer in the present dispersive reflectometer than in the angle-scan reflectometer used at SPring-8. A closer comparison reveals that the reflectivity measured with the present dispersive method is slightly smaller at $Q>0.27 \AA^{-1}$ than that with the angle-scan method, but the reason for this difference is not yet clear. The sample surface looked inhomogeneous and slightly different parts of the sample surface may have been irradiated by X-rays between measurements of the present dispersive and the angle-scan methods.

\subsection{Influence of diffuse scattering to the background intensity}

At a particular point $\left(x_{\mathrm{i}}, y_{\mathrm{i}}\right)$ of the detector surface, in addition to the coplanar (within the scattering plane) diffuse scattering of X-rays incident with a glancing angle $\alpha_{\mathrm{i}}$, a sum of the non-coplanar (out of the scattering plane) diffuse scattering of X-rays incident on the sample with a glancing angle $\alpha_{\mathrm{j}}$ over the angle range from $\alpha_{\mathrm{L}}$ to $\alpha_{\mathrm{H}}$ is also recorded in the present simultaneous multiple angle-wavelength dispersive geometry. The sum of these coplanar and non-coplanar diffuse scattering overlaps with the speculary reflected X-rays and forms the background. On the other hand, in the conventional angle-scan method, only the coplanar diffuse scattering of X-rays incident with a glancing angle $\alpha_{\mathrm{i}}$ overlaps with the specularly reflected beam and forms the background. For the case of the silicon (100) wafer, we compared the intensity profiles along the $y$-axis of the PILATUS detector of curve $a$ (the present dispersive method) in Fig. 5 with that of curve $e$ (the angle-scan method with the $0.1 \mathrm{~mm}$-wide slit). In the case of the present dispersive method, the signal-to-background (S/B) ratio was relatively large $(\mathrm{S} / \mathrm{B}>30)$ at low $Q$, but medium $(\mathrm{S} / \mathrm{B}<\sim 10)$ or less at high $Q\left(>\sim 0.4 \AA^{-1}\right)$. On the other hand, in the angle-dispersive method using a fine slit, the S/B ratio was larger than 80-90 even at high $Q$. This means that the sum of non-coplanar diffuse scattering originating from different $Q$ mainly contributes to the background 
intensity in the present dispersive geometry. Still, we were able to identify the specularly reflected beam surrounded by the broad and slowly varying background, as shown in Fig. 4, at levels of the specular reflectivity in the $10^{-8}$ range. The broad and slowly varying background intensity distribution might be partly due to the averaging effect over different glancing angles $\alpha_{\mathrm{j}}$ of the X-rays and partly due to the nature of the samples used in the present study. We separated the specularly reflected beam intensity from the background intensity using the intensity profile along the $y$-axis of the image recorded by the detector. We also confirmed that the background intensity level of the intensity plot parallel to the $x$-axis is the same as that along the $y$-axis within the statistical errors and that the specular beam intensity derived by subtracting the background in the plot along the $x$-axis of the detector gives almost the same reflectivity curves within the error bars.

The present way of handling the background intensity can be compared with the case of a simultaneous multi-angledispersive X-ray reflectometer using a laboratory X-ray source, a knife edge and a one-dimensional detector (Naudon et al., 1989). Agnihotori \& Ortega (2001) reported that the reflectivity measured with the Naudon-type reflectometer is about five to ten times higher than that measured with the conventional angle-scan reflectometer even in the reflectivity range $10^{-4}-10^{-5}$. It was difficult to separate the specular reflection intensity from the diffuse scattering intensity in their geometry. On the other hand, in the present study the specular reflection intensity is separated from the background and the agreement between reflection curves of the present dispersive method and the angle-scan method is very good down to a reflectivity of $\sim 2 \times 10^{-7}$ for the case of the silicon single crystal and $\sim 3 \times 10^{-5}$ for the case of the gold film.

The present procedure of subtracting the background intensity can be applied to such samples giving a relatively uniform background intensity distribution. For samples which will give a highly non-uniform background intensity distribution, a more careful background-subtraction process would be required such as two-dimensional intensity mapping around the specular reflection. This problem is a subject to be studied in the future.

The measurable minimum reflectivity is lower for less diffusely scattering material. The measured minimum reflectivity was $6.3 \times 10^{-9}$ for the silicon wafer, while that for the ethylene glycol was $4.8 \times 10^{-8}$.

\subsection{Sample space}

It is important that there is enough space around the sample to install chambers or equipment for controlling sample environments or applying a stimulus to the sample. In the present geometry the distance from the focus to both the upstream and downstream slits (not shown in Fig. 1) was $100 \mathrm{~mm}$, giving a free space of cylindrical shape of $200 \mathrm{~mm}$ diameter around the sample. With a more careful alignment of these slits the diameter of this cylinder-shaped free space could be widened to $300 \mathrm{~mm}$.

\subsection{Reflectivity curves from a liquid surface}

In Fig. 7 the agreement between the experimental curve $a$ and the calculated curve $c$ is fairly good, although there are small discrepancies in the $Q$-range of $0.08-0.22 \AA^{-1}$. The S/B ratios in this $Q$-range were approximately 1.1-1.4, because the signal intensity $I=I_{0} \times R$ was very weak as a result of low values of both $I_{0}$ and $R$ as explained in Fig. 3. Such low values of $\mathrm{S} / \mathrm{B}$ could possibly be responsible for the discrepancies. S/B ratios will be improved by increasing the $\mathrm{X}$-ray intensity in this $Q$-range.

The reflectivity curves in Fig. 7 demonstrate that the present reflectometer is suitable for studying liquid surfaces owing to its characteristics that no mechanical movements of the polychromator crystal, slits, sample and detector are required during the measurement while the sample surface is kept in the horizontal plane.

\subsection{Potential for time-resolved measurements}

Since a function to successively record images is already installed in the control software of the PILATUS 100K detector with a $2.8 \mathrm{~ms}$ readout time for each image, timeresolved data collection will easily be conducted with a time resolution of several milliseconds or longer depending on the speed of the structural change of the sample and the X-ray intensity to be measured. Although some example curves shown in Figs. 5, 6 and 7 were obtained in seconds or subseconds, millisecond time resolution will be attained by using focusing mirrors and/or a more intense X-ray source such as multipole wigglers. Especially, the present method will be most suitable for samples which undergo irreversible structural changes because the time-dependent change of the whole profile of the X-ray reflectivity curves can be recorded. If the structural changes are repeatable many times upon a certain stimulus, the pump-probe method could also be applied with the present dispersive method enabling much higher time resolutions.

\section{Summary}

The performance of a simultaneous multiple angle-wavelength dispersive X-ray reflectometer was demonstrated, which can measure the whole profile of a specular X-ray reflectivity curve with no need for rotation of the sample, detector or monochromator (polychromator) crystal during the measurement. In this reflectometer a bent-twisted polychromator crystal is used to produce a convergent X-ray beam having a continuously varying energy (wavelength) and glancing angle to the sample surface as a function of direction. This X-ray beam is incident onto the sample placed horizontally at the focal point. The reflected beam intensity distribution across the beam direction was measured with a twodimensional detector downstream of the specimen.

Examples of static measurements from a silicon singlecrystal wafer and a thin gold layer on a silicon substrate suggest that reflectivity curves with the lowest reflectivity in the range of $10^{-8}$ can be obtained with a data collection time 
of 1.0-10 s. Such a time resolution will be useful in studying slow irreversible changes of surface structures. Milliseconds time resolution will be achieved if the lowest reflectivity of the reflectivity curve remains at $10^{-5}$ or $10^{-6}$ in the required range of perpendicular momentum transfer. Higher time resolutions would be attained by using more intense $\mathrm{X}$-ray sources such as multi-pole wigglers at third-generation synchrotron radiation sources.

The example of ethylene glycol suggested that the present reflectometer would be suitable for studying liquid surfaces since no mechanical movement of the sample and the detector are required during measurements.

In conclusion, the present X-ray reflectometer in the simultaneous multiple angle-wavelength dispersive mode paves the way for time-resolved X-ray reflectometry of various surface structural changes, especially irreversible ones.

The authors thank Professor H. Kawata of the Photon Factory for his continual encouragement and support. They are grateful to Dr Y. Higashi of KEK Mechanical Engineering Center for designing a prototype of a variable-radius crystal bender in the early stage of the present study and Dr K. Hyodo of the Photon Factory for his support on the beamline. They also thank Dr K. Sakurai of the National Institute of Materials Science for supplying the thin gold film sample deposited on a silicon substrate. The experiment was carried out on the beamline AR-NE7A of the Photon Factory under the Photon Factory experimental programs 2009G082 and 2011G068. The research was financially supported by the program (Development of Systems and Technology for Advanced Measurement and Analysis) of the Japan Science and Technology Agency.

\section{References}

Agnihotori, D. K. \& Ortega, R. (2001). Adv. X-ray Anal. 44, 302-307. Bhattacharya, M., Mukherjee, M., Sanyal, M. K., Geue, T., Grenzer, J. \& Pietsch, U. (2003). J. Appl. Phys. 94, 2882-2887.

Daillant, J. \& Gibaud, A. (1999). X-ray and Neutron Reflectivity: Principles and Applications, Lecture Notes in Physics Monographs, Berlin/Heidelberg: Springer.

Garrett, R. F., White, J. W., King, D. J., Dowling, T. L. \& Fulllagar, W. (2001). Nucl. Instrum. Methods Phys. Res. A, 467-468, 998-1000.
Generosi, A., Paci, B., Rossi Albertini, V., Perfetti, P., Pennesi, G., Paoletti, A. M., Rossi, G., Gapobianchi, A. \& Caminiti, R. (2005). Appl. Phys. Lett. 86, 114101.

Gonzalez-Silveria, M., Rodriguez-Viejo, J., Clavaguera-Mora, M. T., Bigault, T. \& Labar, J. L. (2007). Phys. Rev. B, 75, 075419.

Kiessig, H. (1931). Ann. Phys. (Berlin), 402, 715-768; 402, 769788.

Kraft, P., Bergamaschi, A., Broennimann, Ch., Dinapoli, R., Eikenberry, E. F., Henrich, B., Johnson, I., Mozzanica, A., Schlepütz, C. M., Willmott, P. R. \& Schmitt, B. (2009). J. Synchrotron Rad. 16, 368-375.

Matsushita, T., Arakawa, E., Harada, T., Hatano, T., Higashi, Y., Yano, Y. F., Niwa, Y., Inada, Y., Nagano, S. \& Seki, T. (2010). AIP Conf. Proc. 1234, 927-930.

Matsushita, T., Arakawa, E., Niwa, Y., Inada, Y., Hatano, T., Harada, T., Higashi, Y., Hirano, K., Sakurai, K., Ishii, M. \& Nomura, M. (2009). Eur. Phys. J. Special Topics, 167, 113.

Matsushita, T., Inada, Y., Niwa, Y., Ishii, M., Sakurai, K. \& Nomura, M. (2007). J. Phys. Conf. Ser. 83, 012021.

Matsushita, T., Niwa, Y., Inada, Y., Nomura, M., Ishii, M., Sakurai, K. \& Arakawa, E. (2008). Appl. Phys. Lett. 92, 024103.

Naudon, A., Chihab, J., Goudeau, P. \& Mimault, J. (1989). J. Appl. Cryst. 22, 460-464.

Nüske, R., Jurgilaitis, A., Enquist, H., DastjaniFarahani, S., Gaudin, J., Guerin, L., Harb, M., KorffSchmising, C., v, Störmer, M., Wulff, M. \& Larsson, J. (2011). Appl. Phys. Lett. 98, 101909.

Paci, B., Generosi, A., Albertini, V., Perfetti, P., De Bettignies, R., Leroy, J. \& Sentein, C. (2006). Appl. Phys. Lett. 89, 043507.

Paci, B., Generosi, A., Rossi Albertini, V., Perfetti, P., De Bettignies, R., Firon, M., Leroy, J. \& Sentein, C. (2005). Appl. Phys. Lett. 87, 194110.

Parratt, L. G. (1954). Phys. Rev. 95, 359-369.

Richter, A. C., Durbin, M. K., Yu, C. J. \& Dutta, P. (1998). Langmuir, 14, 5980-5983.

Rossi Abertini, V., Generosi, A., Paci, B., Perfetti, P., Pennesi, G., Rossi, G., Gapobianchi, A., Paoletti, A. M. \& Caminiti, R. (2003). Appl. Phys. Lett. 82, 3868-3870.

Tanaka, T. \& Kitamura, H. (2001). J. Synchrotron Rad. 8, 12211228.

Trueb, P., Sobott, B. A., Schnyder, R., Loeliger, T., Schneebeli, M., Kobas, M., Rassool, R. P., Peake, D. J. \& Broennimann, C. (2012). J. Synchrotron Rad. 19, 347-351.

White, J. W., Brown, A. S., Garrett, R. F., King, D. J. \& Dowling, T. L. (1999). Aust. J. Phys. 52, 87-100.

Yano, Y. F., Uruga, T., Tanida, H., Toyokawa, H., Terada, Y., Takagaki, M. \& Yamada, H. (2009). Eur. Phys. J. Special Topics, 167, 101-105.

Yano, Y. F., Uruga, T., Tanida, H., Toyokawa, H., Terada, Y. \& Yamada, H. (2010). J. Synchrotron Rad. 17, 511-516. 